\title{
Seasonal and Monthly Variations of Central Serous Chorioretinopathy and the Association with Choroidal Thickness in Chinese Population
}

\author{
Guodong Liü, Khusbu Keyal\#, Ding Xu*, and Fang Wang* \\ Department of Ophthalmology, Shanghai Tenth People's Hospital Affiliated to Tongji University, School of Medicine, Shanghai, 200072, China \\ "These authors contributed equally
}

*Corresponding authors: Fang Wang, Department of Ophthalmology, Shanghai Tenth People's Hospital Affiliated to Tongji University School of Medicine, Shanghai 200072, China, Fax: 0086-021-66307527; E-mail: Fwang123@yeah.net

Ding Xu, Department of Ophthalmology, Shanghai Tenth People's Hospital Affiliated to Tongji University School of Medicine, Shanghai 200072, China

Received: 15 Aug, 2021 | Accepted: 17 Sep, 2021 | Published: 24 Sep, 2021

Citation: Liu G, Keyal K, Xu D, Wang F (2021) Seasonal and Monthly Variations of Central Serous Chorioretinopathy and the Association with Choroidal Thickness in Chinese Population. J Neurol Neurobiol 7(2): dx.doi.org/10.16966/2379-7150.178

Copyright: @ 2021 Liu G, et al. This is an open-access article distributed under the terms of the Creative Commons Attribution License, which permits unrestricted use, distribution, and reproduction in any medium, provided the original author and source are credited.

\section{Abstract}

Purpose: To study seasonal and monthly variation in Central Serous Chorioretinopathy (CSC) and its possible association with choroidal thickness among Chinese population.

Methods: The single centered research reviewed 362 CSC cases, and the differences in seasonal and monthly variations were analyzed using one-way ANOVA. Subfoveal choroidal thickness was measured using enhanced depth imaging optical coherence tomography, and the association between CSC number and choroidal thickness were analyzed using Spearmen rank correlation.

Results: The highest number of CSC cases was seen in spring $(n=104)$, while the lowest was seen in winter ( $n=75)$. The monthly prevalence of CSC was high in April $(n=38)$, and low in February $(n=16)$. There was significant difference among monthly distribution ( $<<0.001)$, but the difference of seasonal variation was insignificant $(p=0.65)$. Furthermore, the monthly choroidal thickness was associated with the monthly variation of CSC number, and a positive correlation was found between these two parameters $\left(R^{2}=0.651, p=0.022\right)$.

Conclusions: The prevalence of CSC development showed monthly variation, which was highest in April and lowest in February. Corresponding to the monthly variation, the seasonal prevalence of CSC was high in spring and low in winter. The fluctuation of choroidal thickness under environmental conditions might be a contributing factor to the seasonal and monthly variation of CSC cases.

Keywords: Central serous chorioretinopathy; Choroidal thickness; Seasonal and monthly variation

\section{Introduction}

Central Serous Chorioretinopathy (CSC) is a macular condition wherein there is a build-up of fluid under the retina, mainly due to the Retinal Pigment Epithelium (RPE) barrier breakdown and choroidal congestion [1]. Patients who suffer from CSC often report blurred vision, image distortion, a dark area or spot in the central visual field [2]. This condition can be classified either as acute or chronic with the former having duration of less than three months. Men between their 20's and 60's are at a higher risk of developing this condition over other age groups [2]. Other risk factors that predispose a patient from developing CSC are: insomnia, use of steroids, autoimmune disease, type A personality, work shift, sleep apnea, and hypertension [3].

Although the pathogenesis of CSC is not fully understood, the increased choroidal thickness has been widely reported in CSC eyes [4], therefore, CSC is also defined as one of the pachychoroidal diseases $[5,6]$. Most of the CSC cases were unilateral but choroidal thickening was widely reported both in affected and fellow eyes, indicating the contribution of systemic factors on choroidal thickening and CSC development. In the current study, we investigate whether a seasonal and monthly variation of CSC cases exists in Chinese population and the possible correlation of CSC incidence with choroidal thickness under different environmental conditions.

\section{Materials and Methods}

We retrospectively reviewed the medical records of 362 CSC patients who attended the department of ophthalmology at tenth people's hospital, Shanghai from the year of 2012 to 2019. Patients who were diagnosed with CSC in the absence of other fundus abnormalities were included. Those with other fundal conditions such as, sub-retinal blood, optic nerve head pathology, or any other evidences of retinal or choroidal diseases were excluded. The CSC patients with a history of corticosteroid treatment or in pregnancy state were also excluded. This study was conducted according to the tenets of the Declaration of Helsinki, and approved by the institutional review board of Shanghai Tenth People's Hospital.

At the initial examination, each patient underwent a comprehensive ophthalmic examination including the measurement of their Best- 
corrected Visual Acuity (BCVA), slit-lamp biomicroscopy, intraocular pressure, fundus photography and enhanced depth Imaging-optical Coherence Tomography (OCT, Cirrus high-definition 5000). Central choroidal thickness was defined as the average thickness from Bruch membrane to the outside of choroid on the fovea. The final diagnosis was proved by the examinations of Fundus Fluorescein Angiography (FFA) Combined with Indocyanine Green Angiography (ICGA). In each follow-up visit, patients underwent a comprehensive ophthalmologic examination including the measurements of BCVA, fundus photography, and choroidal thickness.

To investigate the seasonal and monthly variation in patients with CSC, we counted the number of patients per month (for monthly distribution of CSC cases) and per season (for seasonal distributions). A year was divided into 4 seasons by defining spring as March, April and May, summer as June, July and August, autumn as September, October and November, winter as December, January and February. To explore the possible relationship between CSC number and choroidal thickness, the monthly choroidal thickness was measured, and the correlation was furtherly analyzed using Spearmen rank correlation.

\section{Statistical Analysis}

The statistical analysis was performed with the Statistical Package for the Social Sciences (SPSS). Differences of seasonal or monthly variation were tested by One-way ANOVA. The association between CSC number and choroidal thickness was acquired using Spearmen rank correlation. Values were presented as the mean \pm standard deviation, the criterion significance was assessed at $\mathrm{P}<0.05$ level.

\section{Results}

\section{Basic information}

A total of 410 eyes from 290 male and 72 females were included in this study. CSC was diagnosed based on the presence of sub-retinal fluid and increased choroidal thickness on OCT images, leaks from RPE on FFA and choroidal capillary dilation on ICGA.

There were 314 patients with unilateral CSC and 48 patients with bilateral CSC. Average age of all the CSC patients was $48.7 \pm 10.2 \mathrm{y}$, with an older age in bilateral CSC patients $(53.4 \pm 9.1 \mathrm{y})$. The detailed information of CSC numbers in each year is shown in table 1 . The highest number of CSC cases was seen in spring $(n=104)$, and the lowest in winter $(n=75)$. However, the difference among the four seasons was insignificant $(\mathrm{p}=0.65$, Figure 1$)$.

In terms of the monthly number, the highest number of CSC cases was seen in April ( $n=38$, Figure 2), and lowest in February $(n=16$,

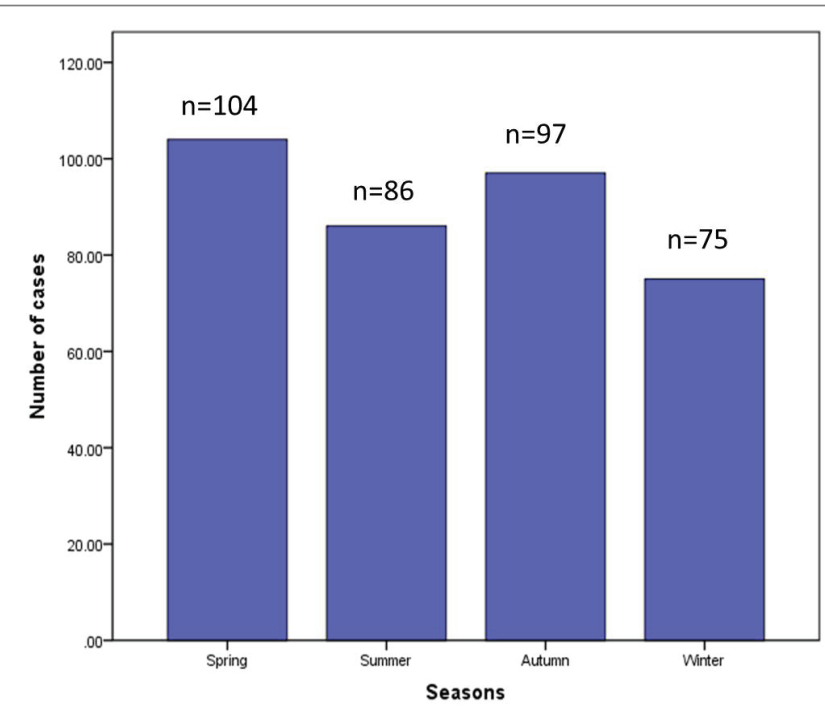

Figure 1: Seasonal distribution of CSC cases. The number of CSC cases was 104 in spring, 86 in summer, 97 in autumn and 75 in winter.

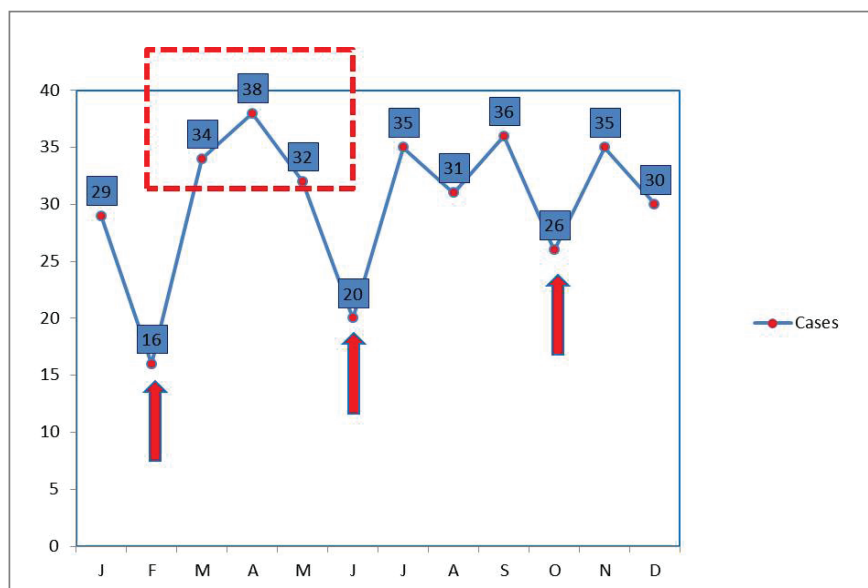

Figure 2: Monthly distribution of CSC cases. The highest number of CSC cases was seen in April ( $n=38)$. There were three troughs (arrows) in the curve, which was the lowest in February within winter $(n=16)$, June within summer $(n=20)$, October within autumn $(n=26)$. And the numbers of CSC cases were generally high in spring (dotted rectangle).

Table 1: Basic information of central serous chorioretinopathy patients in present study.

\begin{tabular}{|c|c|c|c|c|c|c|c|}
\hline Year & $\mathbf{M}$ & $\mathbf{F}$ & Age(Ave) & Unilateral & Age(Ave) & Bilateral & Age(Ave) \\
\hline 2012 & 30 & 8 & $48.3+9.8$ & 31 & $46.6+10.3$ & 7 & $54.1+4.2$ \\
\hline 2013 & 16 & 4 & $51.6+7.9$ & 16 & $49.9+7.9$ & 4 & $57.8+4.5$ \\
\hline 2014 & 37 & 11 & $50.8+10.3$ & 41 & $49+8.5$ & 7 & $66.3+10.6$ \\
\hline 2015 & 39 & 10 & $47.6+10.2$ & 48 & $47.7+10.3$ & 1 & 41 \\
\hline 2016 & 52 & 9 & $46.8+9.6$ & 57 & $46.9+9.6$ & 4 & $47.8+10.4$ \\
\hline 2017 & 54 & 14 & $48.3+10.7$ & 56 & $48.3+11.1$ & 12 & $50.5+9.4$ \\
\hline 2018 & 31 & 6 & $48.3+9.9$ & 27 & $46.5+9.8$ & 10 & $52.9+7.5$ \\
\hline 2019 & 31 & 10 & $49.9+11.1$ & 38 & $49.9+11.5$ & 3 & $53+8.5$ \\
\hline
\end{tabular}

M: Male, F: Female, Ave: Average

Citation: Liu G, Keyal K, Xu D, Wang F (2021) Seasonal and Monthly Variations of Central Serous Chorioretinopathy and the Association with 
Figure 2). Base on the trend to increase or decrease, there were three troughs in the curve, which was February in winter, June in summer and October in autumn respectively. However, the monthly number of CSC cases was generally high in spring (Figure 2).

In terms of the choroidal measurement, the spearman rank correlation revealed a positive correlation between monthly CSC number and choroidal thickness ( $R 2=0.651, \mathrm{p}=0.022$; Figure 3 ). When it comes to the seasonal pattern, the measurements were consistent with the seasonal variation of CSC numbers, which was highest in spring $(426.63 \pm 19.86 \mu \mathrm{m})$, followed by autumn $(388.53$ $\pm 37.23 \mu \mathrm{m})$ and winter $(387.90 \pm 35.75 \mu \mathrm{m})$, and the lowest was found in summer $(377.27 \pm 7.54 \mu \mathrm{m})$ (Figure 3). Similarly, the difference of choroidal thickness among seasons was insignificant $(p=0.176)$. In spite of this, the minimum choroid thickness was found in the month with the less number of CSC cases in each season, which was $362.6 \mu \mathrm{m}$ in February, $383.5 \mu \mathrm{m}$ in June and 348.6 $\mu \mathrm{m}$ in October (Table 2).
Figure 4 shows a CSC eye of a 28 -year-old male with a complaint of blur vision in right eye for two days. Retinal detachment was detected within macular area, where RPE detachment and thickened choroidal thickness were found. The diagnosis of CSC was proved by FFA showing the classic "smokestack" leakage in middle phase.

\section{Discussion}

The current study investigated 362 Chinese patients with the diagnosis of CSC who undergone comprehensive fundus examinations. The aim is to shed light on the seasonal and monthly variation of CSC patients in Chinese population. The study was carried out in Shanghai city located in eastern China with the characteristics of four distinctive seasons.

Our study revealed a seasonal variation of CSC cases, which was highest in spring $(n=104)$ and lowest in winter $(n=75)$. But the difference among the four seasons was insignificant $(\mathrm{p}=0.65$, Figure 1$)$. Nonetheless, the monthly variation was statistically different among

Table 2: Monthly distributions of central serous chorioretinopathy cases and Choroidal Thickness (CT) at foveal center.

\begin{tabular}{|l|c|c|c|c|c|c|c|c|c|c|c|c|}
\hline & \multicolumn{3}{|c|}{ Spring } & \multicolumn{3}{c|}{ Summer } & \multicolumn{3}{c|}{ Autumn } & \multicolumn{3}{c|}{ Winter } \\
\hline Year & M & A & M & J & J & A & S & O & N & D & J & F \\
\hline 2012 & 5 & 2 & 5 & 4 & 3 & 1 & 4 & 5 & 6 & 2 & 1 & 0 \\
\hline 2013 & 0 & 6 & 0 & 1 & 4 & 3 & 2 & 1 & 1 & 1 & 1 & 0 \\
\hline 2014 & 3 & 4 & 2 & 1 & 4 & 7 & 8 & 5 & 9 & 2 & 0 & 2 \\
\hline 2015 & 2 & 6 & 6 & 1 & 5 & 2 & 4 & 1 & 3 & 9 & 11 & 1 \\
\hline 2016 & 12 & 6 & 3 & 3 & 10 & 2 & 5 & 5 & 4 & 1 & 3 & 5 \\
\hline 2017 & 6 & 7 & 6 & 8 & 4 & 9 & 7 & 2 & 8 & 8 & 3 & 1 \\
\hline 2018 & 2 & 5 & 3 & 1 & 2 & 5 & 3 & 4 & 2 & 3 & 5 & 1 \\
\hline 2019 & 4 & 2 & 7 & 1 & 3 & 2 & 3 & 2 & 2 & 4 & 5 & 6 \\
\hline Total & 34 & 38 & 32 & 20 & 35 & 31 & 36 & 25 & 35 & 30 & 29 & 16 \\
\hline CT(um) & 442.9 & 404.5 & 435.5 & 383.5 & 371.2 & 378.3 & 425.3 & 346.8 & 394.7 & 428.8 & 376.2 & 362.6 \\
\hline
\end{tabular}
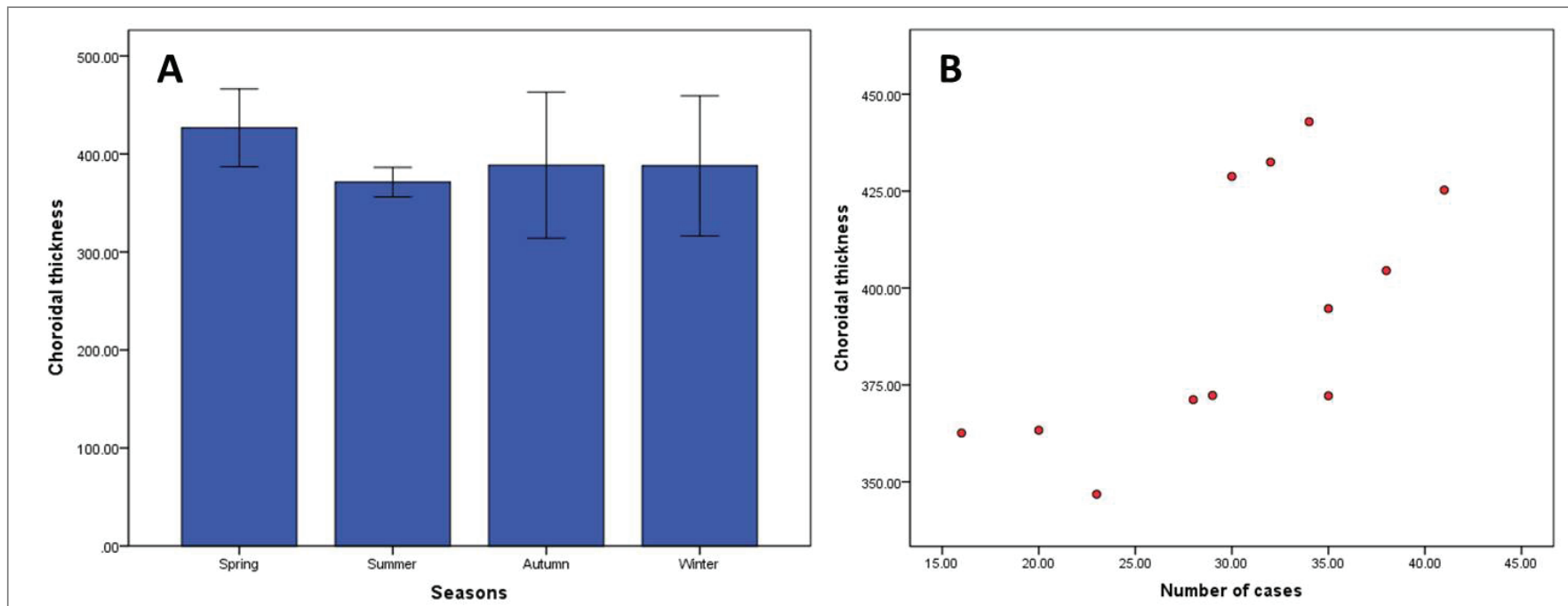

Figure 3: Seasonal distribution of choroidal thickness and correlation with CSC numbers. The thickest choroid were found in spring (426.63 \pm 19.86 um), and the thinnest in summer ( $371.27 \pm 7.54 \mathrm{um}$ ). Spearman rank correlation revealed a positive correlation between CSC number and choroidal thickness $(R=0.651, p=0.022)$. 


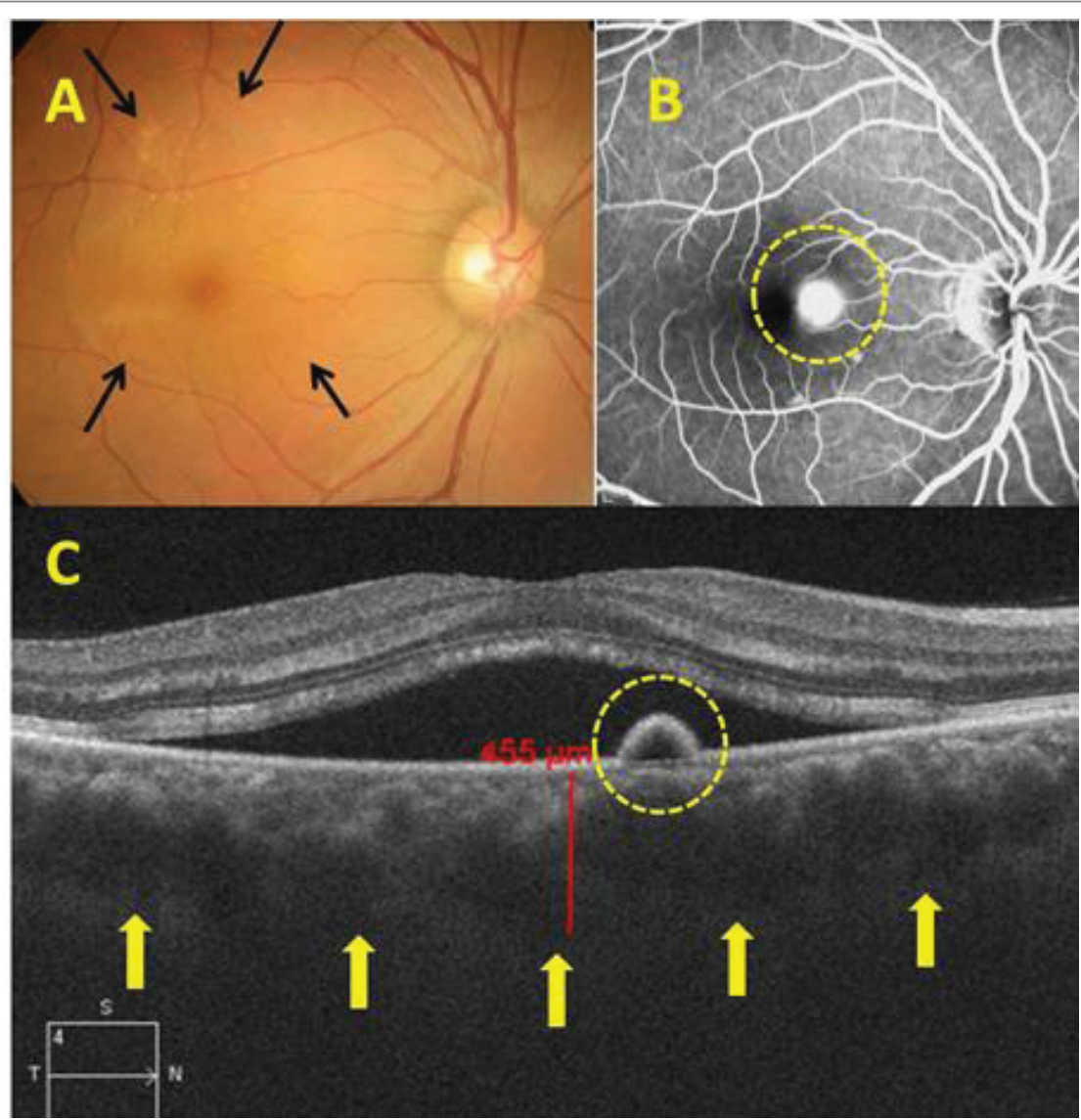

Figure 4: An example of CSC eye from a 28-year-old male. A: Color fundus photograph demonstrates the serous retinal detachment with white exudative spots (black arrows) underling the detached retina. B: Fluorescein angiography shows "smokestack" leakage of dye (dotted circle) in the middle phase. C: Macular OCT reveals serous retinal detachment and RPE detachment (dotted circle) corresponding to the leakage spot on FFA. Central choroidal thickness was increased with a thickness of $455 \mathrm{um}$, and the outside boundary (yellow arrow) could be seen on OCT image.

twelve months, which was highest in April and lowest in February. The distributed pattern was consistent with the results from the only two previous papers that were highest in March among Japanese people [7], and April among American people [8].

In addition to the widely recognized factors leading to CSC, we looked into allergen as a possible trigger to the development of CSC, giving rise to the assumption that there might be higher incidence of CSC in specific season or months when allergens are mostly present $[9,10]$. The significant effect of climatic change on some allergic diseases have been investigated [11,12], which tended to rise at the shift of pollen seasons, mainly in warmer springs and later frosts. Consistent with the study, our results showed distributed peaks of CSC number in spring and autumn. Thus the seasonal variation of certain environmental allergens was proposed to be a factor influencing CSC occurrence.

Furthermore, another finding was the variation of monthly choroidal thickness that was closely related to the numbers of CSC cases. Choroidal vessels dilation is considered as the pathological factor resulting in RPE dysfunction and sub retinal fluid leakage in CSC $[13,14]$. The diurnal changes of the choroidal thickness have been reported $[15,16]$, however there were no investigations of monthly variation of the choroid in normal eyes. Nevertheless, the influence of allergic sensitivity on increased choroidal thickness has been reported
[17], the results in current study also suggested that the changes of choroidal thickness under different seasonal conditions might be the one of the key factors that induces the development of CSC.

Another index that influences the variation of monthly and seasonal CSC incidence was the light absorption. As the most of light energy is absorbed by RPE resulting in the increase of choroidal thickness within sub-macular area [18]. Studies about the diurnal variation of choroidal thickness revealed that sub-macular choroid positively correlated with the daily time exposed to bright light $[19,20]$. This hypothesis could well explain the difference of CSC incidence among countries with varying climate conditions. However our study revealed the thinnest choroidal thickness and lowest CSC cases in summer when the light exposure is longest. Thus we considered the influence of seasons shift on choroidal thickness or CSC cases was much more prominent than bright light.

Since our results supported the idea that CSC incidence varies among different months and seasons, which was choroidal thickness related, the investigation about the seasonal and monthly variation of choroidal thickness in normal subjects was needed.

\section{Conclusions}

We retrospectively investigated the monthly and seasonal prevalence of CSC that the highest monthly number was seen in April

Citation: Liu G, Keyal K, Xu D, Wang F (2021) Seasonal and Monthly Variations of Central Serous Chorioretinopathy and the Association with 
and the peaks of seasonal number were found in spring and autumn. We also revealed a similar variation of choroidal thickness among different months and seasons, which was positively correlated with CSC numbers. So the variation of CSC cases was related to the monthly changes of choroid thickness under different climate conditions.

\section{Funding Details}

This work was supported by the National Natural Science Foundation of China under Grant (No. 81700840).

\section{Disclosure Statement}

The authors have no financial conflicts of interest to report.

\section{References}

1. Daruich A, Matet A, Dirani A, Bousquet E, Zhao M, et al. (2015) Central serous chorioretinopathy: Recent findings and new physiopathology hypothesis. Prog Retin Eye Res 48: 82-118.

2. Rijssen TJV, Dijk EHCV, Yzer S, Ohno-Matsui K, Keunen JEE, et al. (2019) Central serous chorioretinopathy: Towards an evidencebased treatment guideline. Prog Retin Eye Res 73: 100770.

3. Manayath GJ, Ranjan R, Shah VS, Karandikar SS, Saravanan VR, et al. (2018) Central serous chorioretinopathy: Current update on pathophysiology and multimodal imaging. Oman J Ophthalmol 11: 103-112.

4. Yang L, Jonas JB, Wei W (2013) Choroidal vessel diameter in central serous chorioretinopathy. Acta Ophthalmol 91: 358-362.

5. Cheung CMG, Lee WK, Koizumi H, Dansingani K, Lai TYY, et al. (2019) Pachychoroid disease. Eye (Lond) 33: 14-33.

6. Akkaya S (2018) Spectrum of pachychoroid diseases. Int Ophthalmol 38: 2239-2246.

7. Kida T, Kobayashi T, Sato T, Fukumoto M, Ikeda T (2018) Seasonal Variation in Japanese Central Serous Chorioretinopathy. Ophthalmologica 240: 150-156.

8. Cassel GH, Brown GC, Annesley WH (1984) Central serous chorioretinopathy: a seasonal variation? Br J Ophthalmol 68: 724726.
9. Haimovici R, Koh S, Gagnon DR, Lehrfeld T, Wellik S, et al. (2004) Risk factors for central serous chorioretinopathy: a case-control study. Ophthalmology 111: 244-249.

10. Ersoz MG, ArfS, Hocaoglu M, Muslubas IS, Karacorlu M (2019) Patient characteristics and risk factors for central serous chorioretinopathy: an analysis of 811 patients. Br J Ophthalmol 103: 725-729.

11. Barnes CS (2018) Impact of Climate Change on Pollen and Respiratory Disease. Curr Allergy Asthma Rep 18: 59.

12. D'Amato G, Chong-Neto HJ, Ortega OPM, Vitale C, Ansotegui I, et al. (2020) The effects of climate change on respiratory allergy and asthma induced by pollen and mold allergens. Allergy 75: 22192228.

13. Chung YR, Kim JW, Choi SY, Park SW, Kim JH, et al. (2018) subfoveal choroidal thickness and vascular diameter in active and resolved central serous chorioretinopathy. Retina 38: 102-107.

14. Kuroda S, Ikuno Y, Yasuno Y, Nakai K, Usui S, et al. (2013) Choroidal thickness in central serous chorioretinopathy. Retina 33: 302-308.

15. Chakraborty R, Read SA, Collins MJ (2011) Diurnal variations in axial length, choroidal thickness, intraocular pressure, and ocular biometrics. Invest Ophthalmol Vis Sci 52: 5121-5129.

16. Chakraborty R, Read SA, Collins MJ (2012) Monocular myopic defocus and daily changes in axial length and choroidal thickness of human eyes. Exp Eye Res 103: 47-54.

17. Yenigun A, Elbay A, Dogan R, Ozturan O, Ozdemir MH (2017) The effect of allergic rhinitis with positive skin prick test on choroidal thickness. Eur Arch Otorhinolaryngol 274: 2477-2481.

18. Parver LM (1991) Temperature modulating action of choroidal blood flow. Eye (Lond) 5: 181-185.

19. Ulaganathan S, Read SA, Collins MJ, Vincent SJ (2019) Daily axial length and choroidal thickness variations in young adults: Associations with light exposure and longitudinal axial length and choroid changes. Exp Eye Res 189: 107850.

20. Ulaganathan S, Read SA, Collins MJ, Vincent SJ (2019) Influence of seasons upon personal light exposure and longitudinal axial length changes in young adults. Acta Ophthalmol 97: 256-265. 